\title{
Efectos de la fatiga sobre la actividad muscular durante sucesivos test de sentadilla (30 seg)
}

\author{
A.F. SAN JUAN ${ }^{1}$, J. LOUGEDO ${ }^{2}$, R. DOMÍNGUEZ ${ }^{2}$, P. VEIGA-HERREROS ${ }^{2}$, \\ P GARCÍA-FERNÁNDEZ ${ }^{2}$, M.V. GARNACHO-CASTAÑO ${ }^{3}$, MC LOZANO-ESTEVAN ${ }^{2}$, \\ J. GUODEMAR-PÉREZ ${ }^{4}$, J.L. MATÉ-MUÑOZ ${ }^{2}$ \\ ${ }^{1}$ Facultad Ciencias de la Actividad Física y Deporte-INEF, Departamento Salud y Rendimiento Humano, \\ Universidad Politécnica de Madrid \\ ${ }^{2}$ Facultad Ciencias de la Salud, Universidad Alfonso X el Sabio, Villanueva de la Cañada \\ ${ }^{3}$ Escuela Superior Ciencias de la Salud, TecnoCampus Mataró, Universidad Pompeu Fabra \\ ${ }^{4}$ Facultad Ciencias de la Salud, Universidad Camilo José Cela, Villanueva de la Cañada
}

\begin{abstract}
Resumen
Introducción: La fatiga se define como la incapacidad del sistema neuromuscular para mantener un nivel determinado de potencia. La monitorización de la pérdida de altura de salto ha sido utilizada como un indicador de fatiga muscular. La Electromiografia (EMG) es también una herramienta adecuada para determinar la fatiga ya que tiene una alta correlación con las unidades motoras activas (reclutamiento de fibras musculares). El objetivo del presente estudio es evaluar los efectos de la fatiga sobre la actividad muscular en las extremidades inferiores durante sucesivos test de media sentadilla (30 segundos). Métodos: 5 sujetos sanos entrenados participaron en el estudio. Realizaron 2 sesiones de test, un test de potencia máxima de media sentadilla y 4 series de un test de capacidad anaeróbica de media sentadilla (30 seg.). Resultados: Se observaron diferencias significativas $(P=0.002)$ en la comparativa de los efectos inter-sujetos (Vasto lateral vs. Recto femoral). Observamos también diferencias significativas en la comparación por pares $(P<0.001)$. Conclusiones: Observamos cómo la fatiga modifica la activación neuromuscular del vasto lateral y el recto femoral durante la ejecución de 4 test consecutivos de media sentadilla (30 seg.).
\end{abstract}

Palabras clave: Electromiografía, Fatiga, Fuerza, Media sentadilla.

\begin{abstract}
Introduction: Fatigue is defined as an incapacity of the neuromuscular system to maintain a given power level. Monitoring jump height loss during an exercise session has been used as an indicator of muscular fatigue. EMG is also a useful tool to determine fatigue because it has a high correlation with active motor units (muscle fiber recruitment). The objective of this study is to evaluate the fatigue effects over muscle activity in lower extremities during successive test of half squat (30 seconds). Methods: 5 healthy, strength-trained individuals participated in the study. They realized in 2 test sessions one maximal power test in half squat and 4 series of an anaerobic capacity test (30 sec) in half squat. Results: Significant differences (0.002) were observed in inter-subjects effects (Vastus lateralis vs. Rectus femoris). Also in pairs comparation we observe significant differences $(P<0.001)$. Conclusions: We observe how fatigue changes the neuromuscular activation of vastus lateralis and rectus femoris during the execution of 4 consecutive half squat test $(30 \mathrm{sec})$.
\end{abstract}

Keywords: Electromyography, Fatigue, Strength, Half Squat.

Correspondencia:

Alejandro San Juan Ferrer. Facultad Ciencias Actividad Física y Deporte-INEF, Departamento Salud y Rendimiento Humano, Universidad Politécnica de Madrid, C/ Martín Fierro 7, Madrid, CP 28040, España. E-mail: alejandro.sanjuan@upm.es

DOI: $10.5821 /$ sibb.25.1.5385 


\section{Introducción}

La fatiga se define como la incapacidad del sistema neuromuscular para mantener un nivel de potencia determinado [1]. El salto en contra movimiento o countermovement jump (CMJ), es un movimiento que refleja las propiedades contráctiles y de control neuromuscular globales del sistema locomotor [2]. De este modo, la monitorización de la pérdida de altura de salto durante una sesión de ejercicio ha sido utilizada como un indicador de la fatiga muscular. Numerosos estudios han confirmado la pérdida de altura en el CMJ durante varios ejercicios de ejercicio contra resistencias $[3,4,5]$. La electromiografía de superficie ha demostrado también ser una herramienta útil para la valoración de la fatiga ya que tiene una alta correlación con el número de unidades motoras activas (reclutamiento de fibras) $[6,7,8]$. El presente estudio tiene como objetivo evaluar los efectos de la fatiga sobre la actividad muscular de los miembros inferiores durante sucesivos test de sentadilla (30 seg.).

\section{Materiales y Métodos}

En el estudio participaron 5 sujetos varones con una edad de 21,2 $\pm 1,5$ años, altura de $180,2 \pm 6,2 \mathrm{~cm}$ y peso de $80,7 \pm 7,3 \mathrm{~kg}$. Toda la muestra cumplió los criterios de inclusión propuestos por los investigadores que incluían tener una edad comprendida entre 18 y 25 años; una experiencia en el entrenamiento contra resistencias (frecuencia de 3 sesiones semanales) superior a 6 meses; familiarización con el ejercicio de media sentadilla (MS); levantar, al menos, $150 \mathrm{~kg}$ en un test de una repetición máxima (1 RM) en MS; no utilizar ningún suplemento nutricional ni esteroides anabólicos, al menos, en los 6 meses previos; no presentar ningún tipo de alteración cardiovascular, metabólica, pulmonar u ortopédica que pudiese limitar el rendimiento durante el ejercicio. Todos los sujetos fueron reunidos en una sesión en la que se les informó sobre el procedimiento del estudio, además de facilitar un consentimiento informado que firmaron todos los participantes. Durante las 24 horas previas y hasta la finalización del estudio, los sujetos no realizaron ningún tipo de ejercicio complementario al estudio. Además, durante las 2 horas previas a la realización de las pruebas, los sujetos se abstuvieron de fumar, comer y tomar bebidas con cafeína u otro tipo de estimulantes. El estudio cumplió con la declaración de Helsinki sobre investigaciones con seres humanos, siendo aprobado por el comité de ética de la Universidad Alfonso X El Sabio.

Los sujetos acudieron a un total de 2 sesiones de valoración al laboratorio, en una misma franja horaria (+ 2 horas), separadas entre sí por 72 horas. En la $1^{\circ}$ sesión se realizó un Test de potencia máxima en MS. Durante la $2^{\circ}$ sesión, los sujetos realizaron 4 series del Test de capacidad anaeróbica en MS.

Para el Test de potencia máxima en MS, y después de realizar un calentamiento general y otro específico, los sujetos comenzaron con una carga inicial de $20 \mathrm{~kg}$ incrementando la carga en cada serie $10 \mathrm{~kg}$, hasta que la velocidad media obtenida fuese inferior a 0,65 $\mathrm{m} \cdot \mathrm{s}-1$. Entonces la carga era ajustada individualmente para cada sujeto con aumentos entre $5 \mathrm{~kg}-1 \mathrm{~kg}$, con el fin de determinar el valor de $1 \mathrm{RM}$ con la mayor precisión posible. Para velocidades medias mayores a $0,65 \mathrm{~m} \cdot \mathrm{s}^{-1} \mathrm{se}$ realizaban dos intentos con cada carga correspondiente. Para velocidades medias menores a $0,65 \mathrm{~m} \cdot \mathrm{s}^{-1}$ se efectuaba una solo tentativa. El tiempo de recuperación entre cada serie fue de 3 minutos para velocidades medias mayores a $0,65 \mathrm{~m} \cdot \mathrm{s}^{-1}$ y de 5 minutos para velocidades medias menores a $0,65 \mathrm{~m} \cdot \mathrm{s}^{-1}$. La media sentadilla se ejecuta con la barra sobre la porción posterior del deltoides en la parte media del trapecio, realizando una extensión completa de las rodillas desde una posición inicial en el que la rodilla se encontraba con una flexión de $90^{\circ}$ [9]. Para una mayor seguridad en la realización del test, la valoración se realizó en una máquina Smith (Matrix Fitness).

El cálculo de la potencia durante el test de potencia máxima se realizó a través del encoder rotatorio Tendo Weight-lifting Analyzer System (Trencin, Slovak Republic). Este sistema está formado por un cable kevlar que transmite, a través de una interface, instantáneamente la velocidad vertical al software específico de la computadora (Tendo Weight- 
lifting Analyzer 3.0.4). Para calcular la velocidad vertical, el sistema divide todo el rango de movimiento en pequeñas secciones fijas, midiendo el tiempo en cada sección con una precisión de un microsegundo.

En la $2^{a}$ sesión para la medición de la capacidad anaeróbica de las piernas se realiza un test de resistencia intermitente en un ejercicio de MS con una carga correspondiente a la que se obtuvieron los máximos niveles de potencia en el test de potencia máxima en MS. Debido a que el metabolismo glucolítico láctico obtiene sus máximos niveles de potencia en la contribución al metabolismo energético durante el ejercicio, se ha seleccionado un test de 30 segundos de duración a una intensidad con la que los sujetos obtienen sus máximos niveles de potencia. Dicho test de MS (30 seg.), se realizará en 4 series divididos con 3 minutos de recuperación. A los sujetos se les instruyó a que realicen cada repetición a la máxima velocidad posible.

Durante los test se registraron valores electromiográficos de los fascículos del músculo cuádriceps, Vasto Lateral (VL) y Recto Femoral (RF). Se utilizaron pares de electrodos de superficie (Ag-AgCL, diámetro de contacto $6 \mathrm{~mm}$, distancia entre electrodos $3 \mathrm{~cm}$ ). Los electrodos de referencia serán colocados por encima de la espina ilíaca anterosuperior. An-

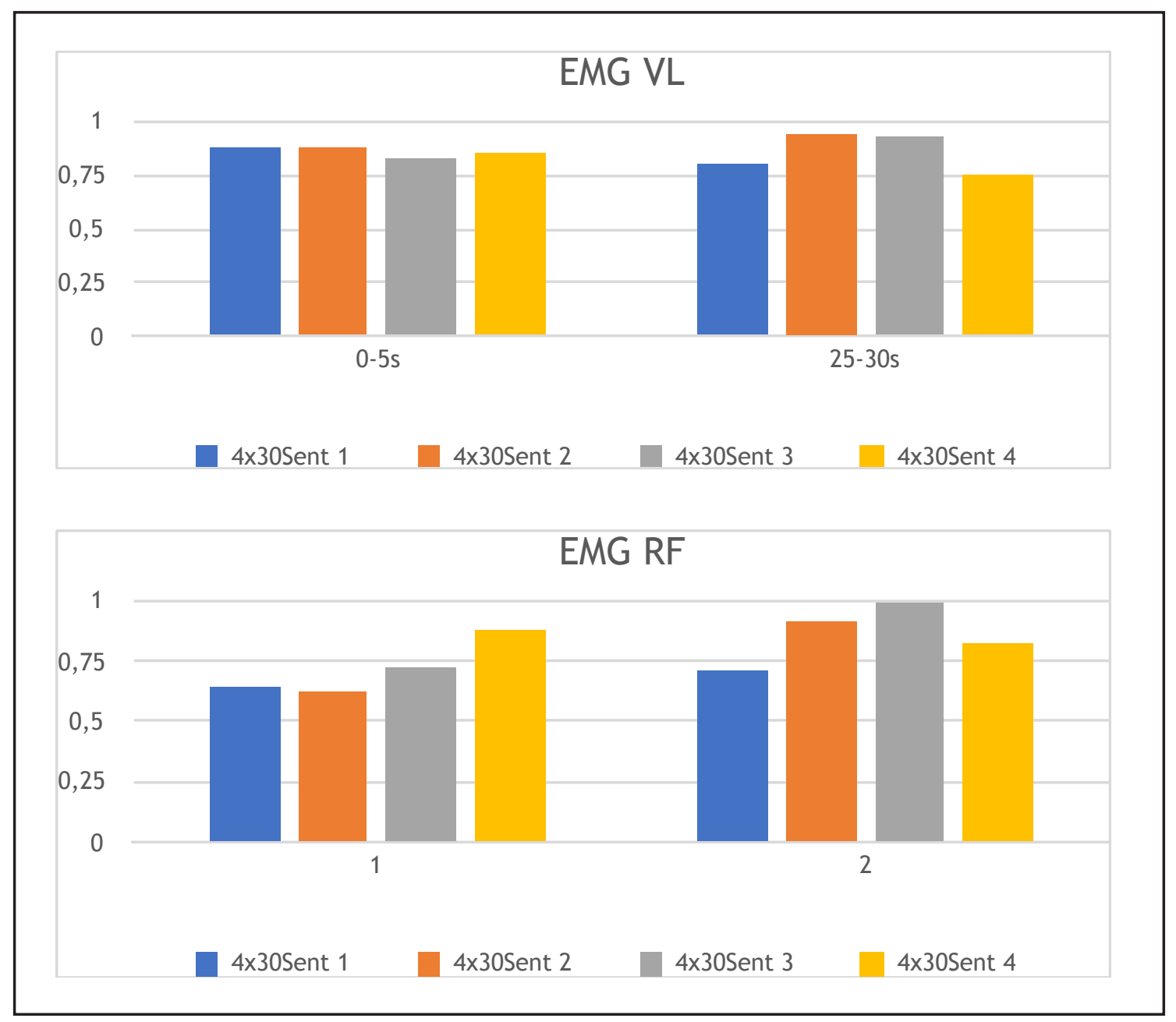

Figura 1. Valores EMG normalizados para cada uno de los 4 test de MS (30 seg.). Comparativa entre el primer grupo de (0-5 seg.) y el último de cada test (25-30 seg.), en VL y RF. 
tes de colocar los electrodos, se procedió a rasurar la zona de piel, a erosionarla ligeramente con papel de lija de grano muy fino y a limpiarla con gasas y alcohol para minimizar la impedancia a menos de $2 \mathrm{k} \Omega$. Los cables conectados a los electrodos fueron bien fijados con esparadrapo para minimizar el posible ruido en la señal producido por los movimientos de los miembros inferiores. La actividad mioeléctrica fue registrada con la ayuda de un analizador de EMG (J\&J Engineering, Poulsbo, WA). La señal EMG-raw fue filtrada por una banda de paso entre 20 y $400 \mathrm{~Hz}$, amplificada y convertida de señal analógica a digital. La variable rms-EMG (root mean square voltaje) obtenida de cada uno de los 2 fascículos, fue normalizada al máximo valor obtenido en el fascículo correspondiente. Con el fin de facilitar el análisis de resultados, se dividieron los 30 segundos de cada test en grupos de 5 segundos, y se evaluaron en el presente trabajo las diferencias entre el primer grupo (0-5 seg.), y el último (25-30 seg.). En el presente estudio la variable rms-EMG fue utilizada para estimar la "actividad mioeléctrica total" del fascículo en ejercicio. Previamente se ha observado que esta computación 1) es una medida precisa de la amplitud de la EMG, y 2) tiene una alta correlación con el número de unidades motoras activas (reclutamiento de fibras) $[6,7,8]$.

En ambas sesiones se realizó un calenta- miento general consistente en 5 minutos de carrera en tapiz rodante (Technogym, Gambettola, Forli, Italia) a una velocidad de 6 $\mathrm{km} \cdot \mathrm{h}-1$, así como de 5 minutos de ejercicios de movilidad articular y estiramientos dinámicos previos, se efectuó un calentamiento específico de una serie de 10 repeticiones de MS con una carga que los sujetos podían desplazar con facilidad. Posteriormente, tras 2 minutos de recuperación se iniciaron los test de la $1^{\text {a }} y$ $2^{a}$ sesión.

El análisis estadístico fue realizado con el software SPSS versión 18.0 (SPSS, Chicago, IL, USA). Se analizó la normalidad de los datos mediante el test de Shapiro-Wilk. El test de Levene reveló la homogeneidad de varianzas de las variables. Para comprobar las diferencias entre las diferentes variables se aplicó un ANOVA de 2 factores de medidas repetidas. Los datos se presentan como media (M) y desviación estándar (DE), y el nivel de significación estadística fue fijado en $\mathrm{p}<0,05$.

\section{Resultados}

En la prueba comparativa de los efectos inter-sujetos (Vasto lateral vs. Recto femoral), la significación es de 0.002 con una diferencia de medias de 0.066. En las comparaciones por pares se observan también diferencias significativas (ver tabla 1).

\begin{tabular}{|l|l|l|l|l|l|}
\hline Test MS & EMG 1 VL & EMG 6 VL & EMG 1 RF & EMG 6 RF & $P$ \\
\hline Test 1 & $0.86 \pm 0.04$ & $0.79 \pm 0.03$ & $0.62 \pm 0.03$ & $0.70 \pm 0.03$ & 0.000 \\
Test 2 & $0.86 \pm 0.04$ & $0.93 \pm 0.03$ & $0.62 \pm 0.02$ & $0.90 \pm 0.03$ & 0.000 \\
Test 3 & $0.82 \pm 0.02$ & $0.92 \pm 0.02$ & $0.71 \pm 0.03$ & $0.99 \pm 0.02$ & 0.000 \\
Test 4 & $0.83 \pm 0.03$ & $0.74 \pm 0.04$ & $0.85 \pm 0.04$ & $0.81 \pm 0.01$ & 0.2 \\
\hline
\end{tabular}

Tabla 1. Valores de EMG normalizados para cada uno de los 4 test de MS (30 seg.). Comparativa entre el primer grupo de (0-5 seg.) y el último de cada test $(25-30 \mathrm{seg}$.). Valores expresados como Media \pm DE. 


\section{Conclusiones}

Durante el presente estudio hemos observado como la fatiga influye en la activación neuromuscular de los músculos vasto lateral y recto femoral del cuádriceps, durante la ejecución de 4 test de MS de 30 segundos consecutivos. Parece que el RF se activa más con la fatiga del VL y participa más en la producción de fuerza. Esto podría estar relacionado con un mayor riesgo de lesión del RF al colaborar en acciones para las que no está estrictamente diseñado en fatiga del VL.

\section{Referencias}

1. Rodacki AL, Fowler NE, Bennett SJ. Multi-segment coordination: fatigue effects. Med Sci Sports Exerc. 2001; 33:1157-67.

2. Bobbert MF; Van Soest AJ. Why do people jump the way they do? Exerc Sport Sci Rev. 2001;29:95102.

3. Rodacki AL, Fowler NE, Bennett SJ. Vertical jump coordination: fatigue effects. Med Sci Sports Exerc. 2002; 34:105-16.
4. Sánchez-Medina L, González-Badillo JJ. Velocity Loss as an indicator of neuromuscular fatigue during resistance training. Med Sci Sports Exerc. 6 2011;43:1725-34.

5. Maté-Muñoz JL, Lougedo JH, Barba M, GarcíaFernández P, Garnacho-Castaño MV, Domínguez R. Muscular fatigue in response to different modalities of CrossFit sessions. PLoS One. 2017:28;12(7):e0181855.

6. Green, H. J. y Patla, A.E. (1992). Maximal aerobic power: neuromuscular and metabolic considerations. Medicine and Science in Sports and Exercise 24, 38-46.

7. Moritani, T., And M. Muro. Motor unit activity and surface electromyogram power spectrum during increasing force of contraction. Eur. J. Appl. Physiol. 56 : 260-265, 1987.

8. Lucia, A., San Juan, A.F., Montilla, M., CaNete, S., Santalla, A., Earnest, C. y Pérez, M. (2004). In professional road cyclists, low pedaling cadences are less efficient. Medicine and Science in Sports and Exercise,36 (6), 1048-1054.

9. Bogdanis GC, Tsoukos A, Veligekas $P$, Tsolakis C, Terzis G. Effects of muscle action type with equal impulse of conditioning activity on postactivation potentiation. J Strength Cond Res. 2014;28(9):2521-8. 